Molecules 2005, 10, 508-515

molecules

ISSN 1420-3049

http://www.mdpi.org

\title{
Microwave-Assisted Amination of a Chloropurine Derivative in the Synthesis of Acyclic Nucleoside Analogues
}

\author{
Andreas Lanver and Hans-Günther Schmalz* \\ Institut für Organische Chemie, Universität zu Köln, Greinstrasse 4, 50939 Köln, Germany \\ Fax: (+49)221-470-3064. \\ * Author to whom correspondence should be addressed; e-mail schmalz@uni-koeln.de \\ Received: 4 January 2005; in revised form: 9 February 2005 / Accepted: 10 February 2005 /
}

Published: 28 February 2005

\begin{abstract}
An efficient protocol for the amination of 6-chloropurine derivatives through nucleophilic aromatic substitution under microwave irradiation was developed and applied to the synthesis in two steps of a series of new acyclic nucleosides (acyclovir analogues) starting from commercially available compounds.
\end{abstract}

Keywords: Microwave, nucleophilic aromatic substitution, nucleosides.

\section{Introduction}

Nucleoside analogues play an important role in pharmacology, mainly as antitumoral or antiviral drugs [1]. Due to their outstanding biological activity various synthetic strategies towards such compounds were developed and have been highlighted in review articles [2]. Prominent examples of synthetic nucleoside analogues are acyclovir (1) [3], a highly active drug against the Herpes Simplex virus, and carbovir (2) [4], an anti-HIV compound. We recently reported on an efficient transitionmetal mediated synthesis of carbocyclic nucleosides such as 3 (Scheme 1), which exhibit significant apoptosis-inducing activities and may therefore be considered as lead structures for the development of new anti-tumoral agents [5]. In any case, the development of efficient protocols for the synthesis of nucleoside analogues (or other privileged classes of molecules) is still an important goal. In an ideal case, only very few steps and short reaction times are necessary to give access to a broad variety of compounds in a rapid and flexible (diversity-oriented) manner. In the past few years, the use of microwave irradiation in organic synthesis has received considerable attention. This technique is of particular value for shortening reaction times and often leads to improved yields [6]. 
Scheme 1. Selected bioactive nucleosides: Acyclovir (1), Carbovir (2), and the apoptosis-inducing nucleoside analog $\mathbf{3}$ (TDS $=$ thexyldimethylsilyl) [5].<smiles>Nc1nc2c(ncn2COCCO)c(=O)[nH]1</smiles>

1

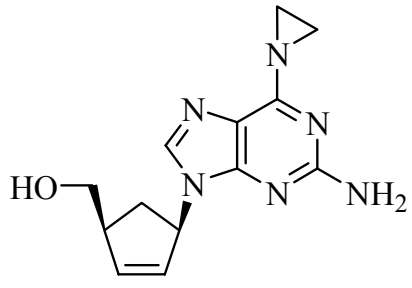

2<smiles>O=S(=O)(O)OCC1CC(n2cnc3c(Cl)ncnc32)C=C1CO</smiles>

3

\section{Results and Discussion}

Intending to exploit these advantages in nucleoside synthesis, we envisioned a two-step reaction sequence towards acyclic nucleoside analogues of type 6, applying a microwave-assisted nucleophilic substitution [7] as a key step (Scheme 2). Following the protocol of Barrio et al. [8], 1.3-dioxolane was first reacted with iodotrimethylsilane in dry cyclohexene at $-78{ }^{\circ} \mathrm{C}$ to afford iodomethyl trimethylsilyloxyethyl ether as an intermediate, which was directly trapped by adding the mixture to a solution of deprotonated chloropurine 4 in THF at $-50{ }^{\circ} \mathrm{C}$. This way, the acyclic nucleoside analogue 5 was obtained in a one-pot procedure.

Scheme 2. Two-step synthesis of acyclic nucleoside analogues of type 6. Conditions: a) 1,3-dioxolane, TMSI, cyclohexene, $-78{ }^{\circ} \mathrm{C}, 30 \mathrm{~min}$; then, addition to $\mathrm{NaH}$ and 4 in DMF, $-50{ }^{\circ} \mathrm{C}$ to RT, $2 \mathrm{~h}$; then, $\mathrm{KF}$ (aq), 54\%.

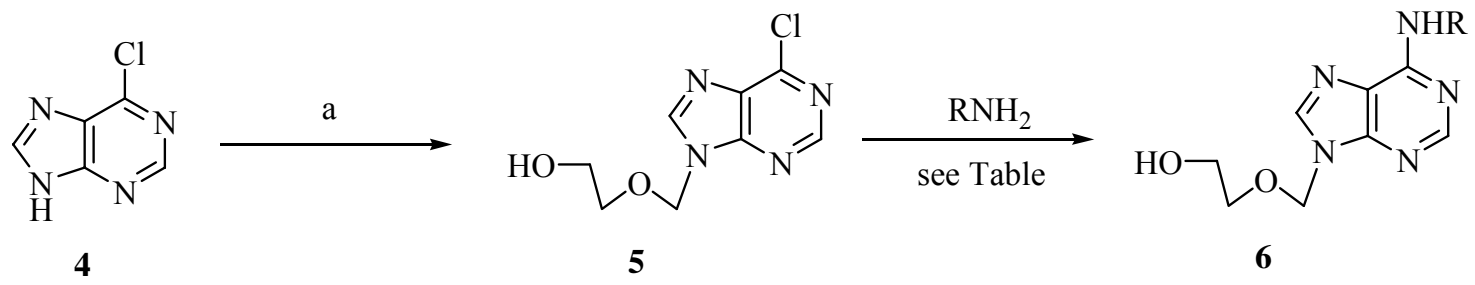

With the acyclovir analogue 5 as a substrate, we carried out a variety of nucleophilic aromatic substitutions using different amines 7-11 as nucleophiles. Yields and reaction times under conventional conditions and under microwave irradiation were compared (Table 1). Our standard protocol for microwave-assisted reactions (CEM single mode $\mathrm{MW}$ reactor, $120{ }^{\circ} \mathrm{C}, 150 \mathrm{~W}$ ) involved $1.1 \mathrm{eq}$ of the corresponding amine, $1.1 \mathrm{eq}$ of diisopropylethylamine (DIPEA) and ethanol as the solvent (conditions A). Alternatively, the reactions were carried out under conventional heating in an oil bath applying established conditions (conditions B and C) for nucleophilic aminations [9]. The microwave assisted reactions proceeded in all cases with higher yields $(72 \%-83 \%)$, and nearly complete conversions were already observed after short reaction times $(10 \mathrm{~min})$. In contrast, much longer reaction times $(16 \mathrm{~h})$ were needed to achieve satisfying conversions under the common conditions (conventional heating), which also led to generally lower yields (58\% - 75\%). 
Table 1. Amination of 5 through nucleophilic substitution.

\begin{tabular}{|c|c|c|c|c|}
\hline Entry & Amine & Product (type 6) & Conditions & Yield \\
\hline 1 & & & A & $82 \%$ \\
\hline 2 & 7 & & $\mathrm{~B}$ & $69 \%$ \\
\hline 3 & & 12 & $\mathrm{C}$ & $72 \%$ \\
\hline 4 & & & A & $77 \%$ \\
\hline 5 & 8 & & B & $70 \%$ \\
\hline 6 & & & $\mathrm{C}$ & $71 \%$ \\
\hline 7 & & & $\mathrm{~A}$ & $83 \%$ \\
\hline 8 & 9 & & B & $75 \%$ \\
\hline 9 & & 14 (lit. [1 & $\mathrm{C}$ & $66 \%$ \\
\hline 10 & & & A & $80 \%$ \\
\hline 11 & 10 & & B & $58 \%$ \\
\hline 12 & & 15 & $\mathrm{C}$ & $65 \%$ \\
\hline 13 & & & A & $72 \%$ \\
\hline
\end{tabular}

Conditions A: 1.1 eq amine, 1.1 eq DIPEA, EtOH, microwave $\left(120^{\circ} \mathrm{C}, 150 \mathrm{~W}\right), 10 \mathrm{~min}$; Conditions B: 1.1 eq amine, 1.1 eq DIPEA, BuOH, $75^{\circ} \mathrm{C}, 16 \mathrm{~h}$; Conditions $\mathrm{C}$ : 5 eq amine, EtOH, $75^{\circ} \mathrm{C}, 16 \mathrm{~h}$.

In our previous work on apoptosis-inducing nucleosides, we observed a positive influence of bulky lipophilic protecting groups (at the 5'-OH group) on the biological activity (e.g. compounds 3) [5]. We therefore converted the key intermediate 5 into protected derivatives in order to study the biological activity of the protected compounds. 
Scheme 3. Synthesis of protected derivatives of 5. Conditions: a) $\mathrm{Ac}_{2} \mathrm{O}, \mathrm{Et}_{3} \mathrm{~N}, \mathrm{DMAP}$, DCM, RT, 1 h, 76\% of 17; b) TDSCl, py, RT, 16 h, 85\% of 18.

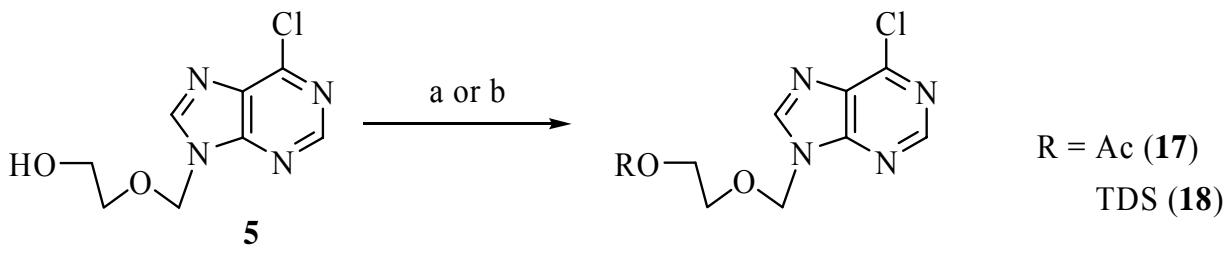

Formation of 17 [11] was accomplished easily under standard acylation conditions $\left(\mathrm{Ac}_{2} \mathrm{O}, \mathrm{Et}_{3} \mathrm{~N}\right.$, DMAP) in DCM. Silylation of 5 also proceeded smoothly with TDSCl (thexyldimethylsilylchloride) in pyridine to give ether $\mathbf{1 8 .}$

\section{Conclusions}

In summary, we have established a microwave-based protocol for the rapid and direct amination of chloropurines in high yield. The value of this method was demonstrated in a synthesis of a series of acyclic nucleoside analogs of type 6. The biological activity (apoptosis induction) of a compound library prepared by the method described is currently under investigation. Furthermore, amination of the protected compounds $\mathbf{1 7}$ and $\mathbf{1 8}$ will be studied.

\section{Acknowledgements}

This work was supported by the "Fond der Chemischen Industrie" (Fonds Stipendium to A.L.). We also thank Dr. H. Schmickler, Dr. N. Schlörer and Dr. M. Schäfer for their support in the application of advanced NMR and MS techniques. Furthermore, we would like to thank Dr. Dr. A. Prokop, Charité Berlin, for the fruitful cooperation concerning the biological evaluation of synthetic nucleosides.

\section{Experimental}

\section{General}

Reagents (generally $\geq 99 \%$ ) were used as purchased. Microwave assisted synthesis was carried out on a CEM focused microwave reactor (model Discover). Reactions were monitored by analytical thinlayer chromatography (TLC) using Merck silica gel 60 F 254 aluminum plates. Chromatograms were visualized either with UV-light, by staining with iodine or with a "cerium reagent" (prepared by dissolving $2 \mathrm{~g}$ of phosphomolybdic acid and $1 \mathrm{~g}$ of $\mathrm{Ce}\left(\mathrm{SO}_{4}\right)_{2}$ in $100 \mathrm{~mL}$ of $10 \%$ aqueous $\left.\mathrm{H}_{2} \mathrm{SO}_{4}\right)$ and subsequent heating. Flash chromatography [12]: silica gel 60 (230-400 mesh) from Merck. Gas chromatography (GLC): HP-6890, with $\mathrm{H}_{2}$ as a carrier gas, FID (flame ionisation detector). NMR: Bruker DPX 300, DRX 500 and AC 250 instruments. Chemical shifts $(\delta)$ are given in ppm relative to the solvent reference. Unless noted otherwise DMSO-D 6 was the solvent used. ${ }^{13} \mathrm{C}$-NMR spectra were measured under proton decoupling and the number of bound protons (multiplicities) determined by DEPT. IR spectroscopy: Perkin Elmer FT-IR Paragon 1000 using the ATR-technique. MS: Finnigan MAT Incos 50 Galaxy System (DIP-MS) or a Finnigan MAT 900 spectrometer; HRMS were recorded on a Finnigan HSQ-30 (HR-EI-MS) or on a Finnigan MAT 900 (HR-ESI-MS). The method of 
ionization is given in parentheses. Melting points (m.p.) were measured on a Büchi B-545 apparatus and are uncorrected.

2-(6-Chloro-purin-9-ylmethoxy)-ethanol (5) [8]: In a first flask, $\mathrm{NaH}$ (55\%, $311 \mathrm{mg}, 7.12 \mathrm{mmol})$ was added to a stirred solution of 6-chloropurine $(1.00 \mathrm{~g}, 6.47 \mathrm{mmol})$ in DMF $(5 \mathrm{~mL})$ at RT and stirring was continued for $2 \mathrm{~h}$. In a second flask, a solution of 1,3-dioxolane (542 $\mu \mathrm{L}, 7.76 \mathrm{mmol})$ in cyclohexene $(2 \mathrm{~mL})$ was cooled to $-78^{\circ} \mathrm{C}$ and TMSI $(1.02 \mathrm{~mL}, 7.12 \mathrm{mmol})$ was added slowly. After $30 \mathrm{~min}$, the first flask was cooled to $-50{ }^{\circ} \mathrm{C}$ (Note: a precipitate was observed at ca. $-60{ }^{\circ} \mathrm{C}$ ) and the contents of the second flask was added. After 2 hours the mixture was allowed to warm to RT and 10\% aqueous solutions of $\mathrm{KF}(10 \mathrm{~mL})$ and $\mathrm{NaHCO}_{3}(10 \mathrm{~mL})$ were added. After $1 \mathrm{~h}$, the mixture was diluted with $\mathrm{H}_{2} \mathrm{O}(50 \mathrm{~mL})$ and extracted with EtOAc $(5 \times 50 \mathrm{~mL})$. The combined organic phases were washed with brine $(50 \mathrm{~mL})$, dried $\left(\mathrm{MgSO}_{4}\right)$ and the solvent was removed under reduced pressure. After flash chromatography (EtOAc/MeOH 10:1) the product 5 was obtained as a light yellow solid (798 mg, $54 \%$ ). (Note: Scale up of the reaction proved to be difficult. In each case a variety of byproducts were obtained, including regioisomers during the amination reactions. The product could still be purified by flash chromatography, but the yields were generally lower). M.p. $147^{\circ} \mathrm{C}$ (lit. [8c]: 149-150 ${ }^{\circ} \mathrm{C}$ ); TLC: $\mathrm{R}_{f}=0.24\left(\right.$ EtOAc/MeOH 10:1); ${ }^{1} \mathrm{H}-\mathrm{NMR}\left(250 \mathrm{MHz}, \mathrm{CDCl}_{3}\right): \delta=8.77(\mathrm{~s}, 1 \mathrm{H}, \mathrm{H}-2), 8.27(\mathrm{~s}, 1 \mathrm{H}, \mathrm{H}-8)$, $5.75\left(\mathrm{~s}, 2 \mathrm{H}, \mathrm{NCH}_{2} \mathrm{O}\right), 3.77-3.66\left(\mathrm{~m}, 4 \mathrm{H}, \mathrm{HOCH}_{2} \mathrm{CH}_{2} \mathrm{O}\right), 1.94(\mathrm{bs}, 1 \mathrm{H}, \mathrm{OH}) ;{ }^{13} \mathrm{C}-\mathrm{NMR}(63 \mathrm{MHz}$, $\left.\mathrm{CDCl}_{3}\right): \delta=152.0$ and 149.1 (C4 and C6), $151.9(\mathrm{C} 2), 147.6(\mathrm{C} 8), 130,7(\mathrm{C} 5), 73.1\left(\mathrm{NCH}_{2} \mathrm{O}\right), 71.1$ $\left(\mathrm{HOCH}_{2} \mathrm{CH}_{2} \mathrm{O}\right), 59.8\left(\mathrm{HOCH}_{2} \mathrm{CH}_{2} \mathrm{O}\right)$; IR (ATR, cm $\left.{ }^{-1}\right): 3378(\mathrm{~m}), 1593(\mathrm{~m}), 1560(\mathrm{~s}), 1335(\mathrm{~m}), 1208$ (m), 1116 (s), 1073 (m), 940 (m), 753 (m), 635 (m); MS (EI, 70 eV): m/z (\%)=229 (13) [M( $\left.{ }^{35} \mathrm{Cl}\right)+$ $1^{+}$], 198 (23), 183 (36), 168 (80), 154 (64), 119 (47), 104 (22); HRMS (EI, $\left.70 \mathrm{eV}\right)$ calcd. for $\left[\mathrm{M}\left({ }^{35} \mathrm{Cl}\right)\right.$ $\left.-\mathrm{CH}_{2} \mathrm{O}\right]^{+} \mathrm{C}_{7} \mathrm{H}_{7}{ }^{35} \mathrm{ClN}_{4} \mathrm{O}: 198.0308$, found: 198.030 .

General protocols for the amination of 5 (compare Table 1): Conditions A: In a sealed microwave tube $5(57.2 \mathrm{mg}, 0.25 \mathrm{mmol})$ was reacted with the amine (1.1 eq, $0.275 \mathrm{mmol})$ and ${ }^{\mathrm{i}} \operatorname{Pr}_{2} \mathrm{NEt}(1.1 \mathrm{eq}$, $0.275 \mathrm{mmol})$ in EtOH $(3 \mathrm{~mL})$ as solvent. The reaction mixture was stirred for $10 \mathrm{~min}$ under microwave irradiation $\left(120^{\circ} \mathrm{C}, 150 \mathrm{~W}\right)$. The solvent was removed under reduced pressure and the crude mixture was directly subjected to flash chromatography (EtOAc/MeOH 10:1). Conditions B: In a Schlenk flask, 5 (57.2 $\mathrm{mg}, 0.25 \mathrm{mmol})$ was reacted with the amine $(1.1 \mathrm{eq}, 0.275 \mathrm{mmol})$ and ${ }^{\mathrm{i}} \operatorname{Pr}_{2} \mathrm{NEt}(1.1 \mathrm{eq}$, $0.275 \mathrm{mmol})$ in $\mathrm{BuOH}(3 \mathrm{~mL})$ as solvent. The reaction mixture was stirred for $16 \mathrm{~h}$ at $75{ }^{\circ} \mathrm{C}$. The solvent was removed under reduced pressure and the crude mixture was directly subjected to flash chromatography (EtOAc/MeOH 10:1). Conditions $C$ : In a Schlenk flask, 5 (57.2 mg, $0.25 \mathrm{mmol}$ ) was reacted with the amine (5 eq, $1.25 \mathrm{mmol})$ in $\mathrm{EtOH}(3 \mathrm{~mL})$ as solvent. The reaction mixture was stirred for $16 \mathrm{~h}$ at $75{ }^{\circ} \mathrm{C}$. The solvent was removed under reduced pressure and the crude mixture was directly subjected to flash chromatography (EtOAc/MeOH 10:1).

2-(6'-Cyclohexylamino-purin-9'-ylmethoxy)-ethanol (12): The acyclic nucleoside analog 12 was prepared according to the general protocols for the amination of 5 using conditions $\mathrm{A}, \mathrm{B}$ or $\mathrm{C}$ (for yields see Table 1). M.p. $102{ }^{\circ} \mathrm{C}$; TLC: $\mathrm{R}_{f}=0.21$ (EtOAc/MeOH 10:1); ${ }^{1} \mathrm{H}-\mathrm{NMR}(250 \mathrm{MHz}): \delta=8.26$ and $8.22\left(2 \mathrm{~s}, 2 \mathrm{H}, \mathrm{H}-2^{\prime}\right.$ and $\left.\mathrm{H}-8^{\prime}\right), 7.53(\mathrm{~d}, \mathrm{~J}=8.4 \mathrm{~Hz}, 1 \mathrm{H}, \mathrm{NH}), 5.56\left(\mathrm{~s}, 2 \mathrm{H}, \mathrm{NCH}_{2} \mathrm{O}\right), 4.67(\mathrm{t}, \mathrm{J}=5.2$ $\mathrm{Hz}, 1 \mathrm{H}, \mathrm{OH}), 3.53-3.45$ (m, 4H, $\left.\mathrm{HOCH}_{2} \mathrm{CH}_{2} \mathrm{O}\right)$ 1.90-1.10 (m, $\left.11 \mathrm{H}, \mathrm{CyHex}\right) ;{ }^{13} \mathrm{C}-\mathrm{NMR}(63 \mathrm{MHz}): \delta=$ 
152.8 (C2'), 153.7 and 149.0 (C4' and C6'), $140.7\left(\mathrm{C}^{\prime}{ }^{\prime}\right), 118.5\left(\mathrm{C}^{\prime}{ }^{\prime}\right), 72.1\left(\mathrm{NCH}_{2} \mathrm{O}\right), 70.7$ $\left(\mathrm{HOCH}_{2} \mathrm{CH}_{2} \mathrm{O}\right), 59.8\left(\mathrm{HOCH}_{2} \mathrm{CH}_{2} \mathrm{O}\right), 48.6(\mathrm{NCH}), 32.3,25.1,24.9(\mathrm{CyH}) ; \mathrm{IR}\left(\mathrm{ATR}, \mathrm{cm}^{-1}\right): 3323(\mathrm{~m})$, 2927 (m), 1615 (s), 1478 (m), 1343 (m), 1293 (m), 1217 (m), 1114 (m), 754 (m); MS (EI, 70 eV): m/z $(\%)=291(8)\left[\mathrm{M}^{+}\right], 216(19), 209$ (50), 174 (24), 160 (26), 148 (26), 135 (100)55 (17); HRMS (EI, 70 EV) calcd. for $\left[\mathrm{M}^{+}\right] \mathrm{C}_{14} \mathrm{H}_{21} \mathrm{~N}_{5} \mathrm{O}_{2}: 291.1695$, found: 291.169 .

2-(6'-Phenylamino-purin-9'-ylmethoxy)-ethanol (13): The acyclic nucleoside analog 13 was prepared according to the general protocols for the amination of 5 using conditions A, B or C (for yields see Table 1). M.p. $169{ }^{\circ} \mathrm{C}$; TLC: $\mathrm{R}_{f}=0.27$ (EtOAc/MeOH 10:1); ${ }^{1} \mathrm{H}-\mathrm{NMR}(250 \mathrm{MHz}): \delta=9.90(\mathrm{~s}, 1 \mathrm{H}$, $\mathrm{NH}), 8.46$ and 8.44 (2s, 2H, H-2' and H-8'), 7.95 (d, J = $7.8 \mathrm{~Hz}, 2 \mathrm{H}, \mathrm{H}_{\mathrm{ar}}$ ), 7.33 (t, J = 7.7 Hz, 2H, $\mathrm{H}_{\mathrm{ar}}$ ), $7.04\left(\mathrm{t}, \mathrm{J}=7.3 \mathrm{~Hz}, 1 \mathrm{H}, \mathrm{H}_{\mathrm{ar}}\right), 5.64\left(\mathrm{~s}, 2 \mathrm{H}, \mathrm{NCH}_{2} \mathrm{O}\right), 4.69(\mathrm{t}, \mathrm{J}=5.3 \mathrm{~Hz}, 1 \mathrm{H}, \mathrm{OH}), 3.58-3.46(\mathrm{~m}, 4 \mathrm{H}$, $\mathrm{HOCH}_{2} \mathrm{CH}_{2} \mathrm{O}$ ); ${ }^{13} \mathrm{C}_{-N M R}(63 \mathrm{MHz}): \delta=152.3\left(\mathrm{C} 2{ }^{\prime}\right), 152.0$ and 149.8 (C4' and C6'), $142.0\left(\mathrm{C}^{\prime}\right)$, $139.5\left(\mathrm{~s}, \mathrm{C}_{\mathrm{ar}}\right), 128.3,122.5,120.7\left(\mathrm{~d}, \mathrm{C}_{\mathrm{ar}}\right), 119.5(\mathrm{C} 5), 72.3\left(\mathrm{NCH}_{2} \mathrm{O}\right), 70.8\left(\mathrm{HOCH}_{2} \mathrm{CH}_{2} \mathrm{O}\right), 59.8$ $\left(\mathrm{HOCH}_{2} \mathrm{CH}_{2} \mathrm{O}\right)$; IR (ATR, cm ${ }^{-1}$ ): 3322 (m), 1621 (s), 1580 (s), 1496 (m), 1477 (m), 1115 (m), 753

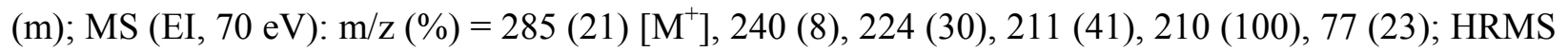
(EI, $70 \mathrm{eV}$ ) calcd. for $\left[\mathrm{M}^{+}\right] \mathrm{C}_{14} \mathrm{H}_{15} \mathrm{~N}_{5} \mathrm{O}_{2}$ : 285.1226, found: 285.122 .

2-(6'-Benzylamino-purin-9'-ylmethoxy)-ethanol (14) [10]: The acyclic nucleoside analog 14 was prepared according to the general protocols for the amination of $\mathbf{5}$ using conditions $\mathrm{A}, \mathrm{B}$ or $\mathrm{C}$ (for yields see Table 1). M.p. $111{ }^{\circ} \mathrm{C}$ (lit. [10]: 113-114 ${ }^{\circ} \mathrm{C}$ ); TLC: $\mathrm{R}_{f}=0.22$ (EtOAc/MeOH 10:1); ${ }^{1} \mathrm{H}-\mathrm{NMR}(250 \mathrm{MHz}): \delta=8.38(\mathrm{~s}, 1 \mathrm{H}, \mathrm{NH}), 8.30$ and $8.24\left(2 \mathrm{~s}, 2 \mathrm{H}, \mathrm{H}-2{ }^{\prime}\right.$ and $\left.\mathrm{H}-8^{\prime}\right), 7.36-7.18(\mathrm{~m}, 5 \mathrm{H}$, $\left.\mathrm{H}_{\mathrm{ar}}\right), 5.58\left(\mathrm{~s}, 2 \mathrm{H}, \mathrm{NCH}_{2} \mathrm{O}\right), 4.72\left(\mathrm{~s}, 2 \mathrm{H}, \mathrm{NCH}_{2} \mathrm{C}_{\mathrm{ar}}\right), 4.67(\mathrm{t}, \mathrm{J}=5.2 \mathrm{~Hz}, 1 \mathrm{H}, \mathrm{OH}), 3.54-3.46(\mathrm{~m}, 4 \mathrm{H}$, $\mathrm{HOCH}_{2} \mathrm{CH}_{2} \mathrm{O}$ ); ${ }^{13} \mathrm{C}^{-\mathrm{NMR}}$ (63 MHz): $\delta=154.3$ and 152.7 (C4' and $\mathrm{C}^{\prime}$ '), 154.3 (C2'), 141.0 (C8'), $\left.139.9\left(\mathrm{~s}, \mathrm{C}_{\mathrm{ar}}\right), 128.1,127.0,126.5\left(\mathrm{~d}, \mathrm{C}_{\mathrm{ar}}\right), 118.8(\mathrm{C})^{\prime}\right), 72.2\left(\mathrm{NCH}_{2} \mathrm{O}\right), 70.7\left(\mathrm{HOCH}_{2} \mathrm{CH}_{2} \mathrm{O}\right), 59.8$ $\left(\mathrm{HOCH}_{2} \mathrm{CH}_{2} \mathrm{O}\right), 42.8\left(\mathrm{NCH}_{2} \mathrm{C}_{\mathrm{ar}}\right)$; IR (ATR, cm $\left.{ }^{-1}\right): 3283(\mathrm{~m}), 2926(\mathrm{~m}), 1616(\mathrm{~s}), 1482(\mathrm{~m}), 1343(\mathrm{~m})$, $1217(\mathrm{~m}), 1114(\mathrm{~m}), 1073(\mathrm{~m}), 761(\mathrm{~m}), 645$ (m); MS (EI, $70 \mathrm{eV}): \mathrm{m} / \mathrm{z}(\%)=299(39)\left[\mathrm{M}^{+}\right], 254(8)$, 238 (16), 225 (66), 224 (91), 120 (26), 106 (95), 91 (100), 79 (16), 65 (25); HRMS (EI, 70 eV) calcd. for $\left[\mathrm{M}^{+}\right] \mathrm{C}_{15} \mathrm{H}_{17} \mathrm{~N}_{5} \mathrm{O}_{2}$ : 299.1382, found: 299.138 .

2-[6'-(p-Morpholinyl-phenylamino)-purin-9'-ylmethoxy]-ethanol (15): The acyclic nucleoside analog $\mathbf{1 5}$ was prepared according to the general protocols for the amination of $\mathbf{5}$ using conditions A, B or C (for yields see Table 1). M.p. $186{ }^{\circ} \mathrm{C}$; TLC: $\mathrm{R}_{f}=0.16$ (EtOAc/MeOH 10:1); ${ }^{1} \mathrm{H}-\mathrm{NMR}(250 \mathrm{MHz}): \delta=$ 9.69 (s, 1H, NH), 8.41 and 8.36 (2s, 2H, H-2' and H-8'), 7.74 (d, J = 9.0 Hz, 2H, $\left.\mathrm{H}_{\mathrm{ar}}\right), 6.92$ (d, J = 9.0 $\left.\mathrm{Hz}, 2 \mathrm{H}, \mathrm{H}_{\mathrm{ar}}\right), 5.62\left(\mathrm{~s}, 2 \mathrm{H}, \mathrm{NCH}_{2} \mathrm{O}\right), 4.68(\mathrm{t}, \mathrm{J}=5.3 \mathrm{~Hz}, 1 \mathrm{H}, \mathrm{OH}), 3.74\left(\mathrm{t}, \mathrm{J}=4.8 \mathrm{~Hz}, 4 \mathrm{H}, \mathrm{NCH}_{2} \mathrm{CH}_{2} \mathrm{O}\right)$, 3.56-3.45 (m, 4H, $\left.\mathrm{HOCH}_{2} \mathrm{CH}_{2} \mathrm{O}\right), 3.06\left(\mathrm{t}, \mathrm{J}=4.8 \mathrm{~Hz}, 4 \mathrm{H}, \mathrm{NCH}_{2} \mathrm{CH}_{2} \mathrm{O}\right) ;{ }^{13} \mathrm{C}-\mathrm{NMR}(63 \mathrm{MHz}): \delta=$ 152.4 (C2'), 152.1 and 149.5 (C4' and C6'), $147.0\left(\mathrm{~s}, \mathrm{C}_{\mathrm{ar}}\right), 141.6\left(\mathrm{C} 8^{\prime}\right), 131.6\left(\mathrm{~s}, \mathrm{C}_{\mathrm{ar}}\right), 122.2\left(\mathrm{~d}, \mathrm{C}_{\mathrm{ar}}\right)$, $119.2\left(\mathrm{C}^{\prime}\right), 115.2\left(\mathrm{~d}, \mathrm{C}_{\mathrm{ar}}\right), 72.3\left(\mathrm{NCH}_{2} \mathrm{O}\right), 70.8 \quad\left(\mathrm{HOCH}_{2} \mathrm{CH}_{2} \mathrm{O}\right), 66.0 \quad\left(\mathrm{NCH}_{2} \mathrm{CH}_{2} \mathrm{O}\right), 59.8$ $\left(\mathrm{HOCH}_{2} \mathrm{CH}_{2} \mathrm{O}\right), 49.0\left(\mathrm{NCH}_{2} \mathrm{CH}_{2} \mathrm{O}\right)$; IR (ATR, cm $\left.{ }^{-1}\right): 3323(\mathrm{~m}), 2922(\mathrm{~m}), 1618$ (s), $1583(\mathrm{~m}), 1511$ (s), 1477 (m), 1230 (m), 1117 (m), 926 (m), 758 (m); MS (EI, 70 eV): m/z (\%) = 270 (100) [M $\left.{ }^{+}\right], 309$

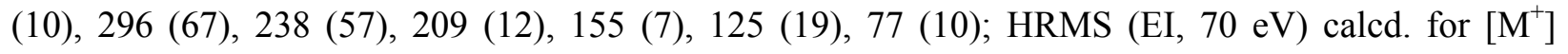
$\mathrm{C}_{18} \mathrm{H}_{22} \mathrm{~N}_{6} \mathrm{O}_{3}: 370.1753$, found: 370.175 . 
2-\{6'-[2-(1''H-Indol-2''-yl)-ethylamino]-purin-9'-ylmethoxy\}-ethanol (16): The acyclic nucleoside analog 16 was prepared according to the general protocols for the amination of 5 using conditions A, B or C (for yields see Table 1). M.p. $147{ }^{\circ} \mathrm{C}$; TLC: $\mathrm{R}_{f}=0.17$ (EtOAc/MeOH 10:1); ${ }^{1} \mathrm{H}-\mathrm{NMR}$ (250 MHz): $\delta=10.80\left(\mathrm{~s}, 1 \mathrm{H}, \mathrm{H}-1^{\prime}\right.$ ') $), 8.31$ and $8.28\left(2 \mathrm{~s}, 2 \mathrm{H}, \mathrm{H}-2\right.$ ' and $\left.\mathrm{H}-8^{\prime}\right), 7.87\left(\mathrm{~s}, 1 \mathrm{H}, \mathrm{CH}_{2} \mathrm{NH}\right), 7.65(\mathrm{~d}, \mathrm{~J}=7.5$ Hz, 1H, H-7' '), 7.35 (d, J = 7.8 Hz, 1H, H-4' '), 7.21 (s, 1H, H-2''), 7.10-6.96 (m, 2H, H-5' and H6"'), $5.58\left(\mathrm{~s}, 2 \mathrm{H}, \mathrm{NCH}_{2} \mathrm{O}\right), 4.68(\mathrm{bs}, 1 \mathrm{H}, \mathrm{OH}), 3.56-3.47\left(\mathrm{~m}, 6 \mathrm{H}, \mathrm{HOCH}_{2} \mathrm{CH}_{2} \mathrm{O}\right.$ and $\left.\mathrm{NCH}_{2} \mathrm{CH}_{2}\right), 3.04$ (t, $\left.\mathrm{J}=8.0 \mathrm{~Hz}, 2 \mathrm{H}, \mathrm{NCH}_{2} \mathrm{CH}_{2}\right) ;{ }^{13} \mathrm{C}-\mathrm{NMR}(63 \mathrm{MHz}): \delta=154.4$ and 148.9 (C4' and $\mathrm{C} 6$ '), 152.8 (C2'), 140.9 (C8'), 136.2 (C3a'), 127.2 (C7a'), 122.5 (C2'), 120.8 (C5'), 118.8 (C5), 118.4 and 118.1 (C4' and C6'), 111.8 (C3'), 111.2 (C7'), $72.2\left(\mathrm{NCH}_{2} \mathrm{O}\right), 70.7 \quad\left(\mathrm{HOCH}_{2} \mathrm{CH}_{2} \mathrm{O}\right), 59.8$ $\left(\mathrm{HOCH}_{2} \mathrm{CH}_{2} \mathrm{O}\right), 48.5\left(\mathrm{NCH}_{2} \mathrm{CH}_{2} \mathrm{C}_{\mathrm{ar}}\right), 25.1\left(\mathrm{NCH}_{2} \mathrm{CH}_{2} \mathrm{C}_{\mathrm{ar}}\right)$; IR (ATR, cm $\left.{ }^{-1}\right): 3284(\mathrm{~m}), 2924(\mathrm{~m}), 1619$ (s), 1454 (m), 1337 (m), 1223 (m), 1112 (m), 1072 (m), 742 (m); MS (ESI): m/z (\%) = 727 (9) [2M + $\left.\mathrm{Na}^{+}\right], 449$ (10), 375 (100) $\left[\mathrm{M}+\mathrm{Na}^{+}\right], 353(50)\left[\mathrm{M}+\mathrm{H}^{+}\right], 279$ (12); HRMS (ESI) calcd. for [M + $\left.\mathrm{H}^{+}\right]$ $\mathrm{C}_{18} \mathrm{H}_{21} \mathrm{~N}_{6} \mathrm{O}_{2}: 353.1725$, found: 353.172 .

2-(6-chloro-purin-9-ylmethoxy)-ethyl acetate (17) [11]: $\mathrm{Ac}_{2} \mathrm{O}(275 \mu \mathrm{L}, 2.88 \mathrm{mmol})$ was added at RT to a stirred solution of $5(300 \mathrm{mg}, 1.31 \mathrm{mmol}), \mathrm{Et}_{3} \mathrm{~N}(235 \mu \mathrm{L}, 1.57 \mathrm{mmol})$ and DMAP (20 mg, 0.16 mmol) in $\mathrm{CH}_{2} \mathrm{Cl}_{2}(8 \mathrm{~mL})$. The resulting mixture was stirred for $1 \mathrm{~h}$ at $\mathrm{RT}$ and was then quenched by addition of sat. aq. $\mathrm{NaHCO}_{3}(10 \mathrm{~mL})$. The water phase was extracted with $\mathrm{CH}_{2} \mathrm{Cl}_{2}(3 \times 10 \mathrm{~mL})$ and the combined organic layers were washed with sat. aq. $\mathrm{NaHCO}_{3}(2 \times 10 \mathrm{~mL})$ and brine $(10 \mathrm{~mL})$, dried $\left(\mathrm{MgSO}_{4}\right)$ and concentrated under reduced pressure. The residue was purified by flash chromatography (EtOAc) to give the product 17 as a white solid (270 mg, 76\%). M.p. $96{ }^{\circ} \mathrm{C}$ (lit. [11b]: 96-97 ${ }^{\circ} \mathrm{C}$ ); TLC: $\mathrm{R}_{f}=0.44$ (EtOAc); ${ }^{1} \mathrm{H}-\mathrm{NMR}(250 \mathrm{MHz}): \delta=8.85(\mathrm{~s}, 1 \mathrm{H}, \mathrm{H}-2), 8.82(\mathrm{~s}, 1 \mathrm{H}, \mathrm{H}-8), 5.73(\mathrm{~s}, 2 \mathrm{H}$, $\left.\mathrm{NCH}_{2} \mathrm{O}\right), 4.08-4.05$ and 3.77-3.74 (m, $\left.4 \mathrm{H}, \mathrm{HOCH}_{2} \mathrm{CH}_{2} \mathrm{O}\right), 1.90\left(\mathrm{~s}, 3 \mathrm{H}, \mathrm{CH}_{3}\right) ;{ }^{13} \mathrm{C}-\mathrm{NMR}(63 \mathrm{MHz}): \delta=$ $170.0(\mathrm{C}=\mathrm{O}), 152.0$ and 149.1 (C4 and $\mathrm{C} 6), 151.9(\mathrm{C} 2), 147.6(\mathrm{C} 8), 130.8(\mathrm{C} 5), 72.8\left(\mathrm{NCH}_{2} \mathrm{O}\right), 67.2$ $\left(\mathrm{HOCH}_{2} \mathrm{CH}_{2} \mathrm{O}\right), 62.6\left(\mathrm{HOCH}_{2} \mathrm{CH}_{2} \mathrm{O}\right), 20.4\left(\mathrm{CH}_{3}\right)$; IR (ATR, cm $\left.{ }^{-1}\right): 2950$ (s), 1735 (s), $1592(\mathrm{~m}), 1560$ (s), 1335 (m), 1234 (s), 1141 (m), 1102 (m), 1049 (m), 938 (m), 752 (m), 636 (m); MS (EI, 70 eV): $\mathrm{m} / \mathrm{z}(\%)=270(5)[\mathrm{M}]^{+}, 259$ (19), 210 (9), 183 (31), 197 (92), 154 (12), 104 (13), 87 (100); HRMS (EI, $70 \mathrm{eV}$ ) calcd. for $[\mathrm{M}]^{+} \mathrm{C}_{10} \mathrm{H}_{11} \mathrm{ClN}_{4} \mathrm{O}_{3}: 270.0520$, found: 270.052 .

6-Chloro-9-\{2-[dimethyl-(1,1,2-trimethyl-propyl)-silanyloxy]-ethoxymethyl\}-9H-purine (18): Chloropurine 5 (300 $\mathrm{mg}, 1.31 \mathrm{mmol})$ was added to dry pyridine $(2.5 \mathrm{~mL})$ at $\mathrm{RT}$ and the mixture was stirred for $5 \mathrm{~min}$. $\mathrm{ThxMe}_{2} \mathrm{SiCl}(409 \mu \mathrm{L}, 1.97 \mathrm{mmol})$ was added and after stirring was continued for $16 \mathrm{~h}$, the reaction was quenched by addition of sat. aq. $\mathrm{NaHCO}_{3}(10 \mathrm{~mL})$. The resulting mixture was stirred at RT for $30 \mathrm{~min}$, then the water phase was extracted with EtOAc $(4 \times 10 \mathrm{~mL})$. The combined organic layers were washed with water $(10 \mathrm{~mL})$ and brine $(10 \mathrm{~mL})$, dried $\left(\mathrm{MgSO}_{4}\right)$ and concentrated under reduced pressure. The residue was then purified by flash chromatography ( $c$-Hex/EtOAc $2: 1)$, giving 18 as a white solid (412 mg, 85\%). M.p. $58{ }^{\circ} \mathrm{C}$; TLC: $\mathrm{R}_{f}=0.18$ (c-Hex/EtOAc 2:1); ${ }^{1} \mathrm{H}-\mathrm{NMR}(250$ $\mathrm{MHz}): \delta=8.84$ and $8.82(2 \mathrm{~s}, 2 \mathrm{H}, \mathrm{H}-2$ and $\mathrm{H}-8), 5.72\left(\mathrm{~s}, 2 \mathrm{H}, \mathrm{NCH}_{2} \mathrm{O}\right), 3.62\left(\mathrm{~s}, 4 \mathrm{H}, \mathrm{OCH}_{2} \mathrm{CH}_{2} \mathrm{O}\right)$, 1.45 (septet, $\left.J=6.8 \mathrm{~Hz}, 1 \mathrm{H}, \mathrm{Me}_{2} \mathrm{CH}\right), 0.73\left(\mathrm{~d}, J=6.8 \mathrm{~Hz}, 6 \mathrm{H},\left(\mathrm{CH}_{3}\right)_{2} \mathrm{CH}\right), 0.69\left(\mathrm{~s}, 6 \mathrm{H}, \mathrm{C}\left(\mathrm{CH}_{3}\right)_{2}\right),-0.03$ (s, 6H, $\left.\left(\mathrm{CH}_{3}\right)_{2} \mathrm{Si}\right) ;{ }^{13} \mathrm{C}-\mathrm{NMR}(63 \mathrm{MHz}): \delta=152.0$ and 149.1 (C4 and $\left.\mathrm{C} 6\right), 151.8(\mathrm{C} 2), 147.5(\mathrm{C} 8)$, $130.8(\mathrm{C} 5), 73.1\left(\mathrm{NCH}_{2} \mathrm{O}\right), 70.9\left(\mathrm{HOCH}_{2} \mathrm{CH}_{2} \mathrm{O}\right), 61.4\left(\mathrm{HOCH}_{2} \mathrm{CH}_{2} \mathrm{O}\right), 33.5\left(\mathrm{Me}_{2} \mathrm{CH}\right), 24.4\left(\mathrm{Me}_{2} \mathrm{CSi}\right)$, $20.0\left(\left(\mathrm{CH}_{3}\right)_{2} \mathrm{CH}\right), 18.1\left(\left(\mathrm{CH}_{3}\right)_{2} \mathrm{CSi}\right),-3.6\left(\left(\mathrm{CH}_{3}\right)_{2} \mathrm{Si}\right)$; IR (ATR, $\left.\mathrm{cm}^{-1}\right): 2955(\mathrm{~m}), 2866(\mathrm{~m}), 1592(\mathrm{~m})$, 
1560 (s), 1251 (m), 1208 (m), 1141 (m), 1096 (s), 937 (m), 830 (s), 777 (m); MS (EI, 70 eV): m/z (\%) $=285(8)\left[\mathrm{M}-\mathrm{C}_{6} \mathrm{H}_{13}{ }^{+}\right], 169$ (33), 167 (100), 117 (5), 104 (6), 73 (12); HRMS (ESI) calcd. for [M $\left.\mathrm{C}_{6} \mathrm{H}_{13}{ }^{+}\right] \mathrm{C}_{10} \mathrm{H}_{14} \mathrm{ClN}_{4} \mathrm{O}_{2} \mathrm{Si}: 285.0574$, found: 285.057 .

\section{References and Notes}

1. a) El Kouni, M. H. Curr. Pharm. Des. 2002, 8, 581-593; b) Richman, D. D. Nature 2001, 410, 995-1001; c) Larder, B. A.; Stammers, D. K. Nat. Struct. Biol. 1999, 6, 103-106; d) De Clerq, E. Nucleos. Nucleot. Nucl. Acids 1994, 12, 1271; e) Sandstrom, E.; Oberg, B. Drugs 1993, 45, 637653; see also: f) Beausejour, C. M.; Gagnon, J.; Primeau, M.; Momparler, R. L. Biochem. Biophys. Res. Commun. 2002, 293, 1478-1484; g) Tjarsk, W. J. Organomet. Chem. 2000, 614-615, 37-74.

2. a) Zhu, X.-F. Nucleos. Nucleot. Nucl. Acids 2000, 19, 651-690: For a recent review on Pd-assisted routes to nucleosides, see: b) Agrofoglio, L. A.; Gillaizeau, I.; Saito, Y. Chem. Rev. 2003, 103, 1875-1916.

3. Gao, H.; Mitra, A. K. Synthesis 2000, 329-351.

4. a) Vince, R.; Hua, M. J. Med. Chem. 1990, 33, 17-21; b) Parker, W. B.; Shaddix, S. C.; Bowdon, B. J.; Rose, L. M.; Vince, R.; Shannon, W. N.; Bennett, L. L. Antimicrob. Agents Chemother. 1993, 37, 1004-1009.

5. a) Velcicky, J.; Lex, J.; Schmalz, H.-G. Org. Lett. 2002, 4, 565-568; b) Velcicky, J.; Lanver, A.; Lex, J.; Prokop, A.; Wieder, Th.; Schmalz, H.-G. Chem. Eur. J. 2004, 10, 5087-5110.

6. For an excellent recent review on microwave-assisted organic synthesis, see: Kappe, C. O. Angew. Chem. 2004, 116, 6408-6443; Angew. Chem. Int. Ed. 2004, 43, 6250-6284.

7. For recent examples on microwave-assisted aminations, see: a) Xu, G.; Wang, Y.-G. Org. Lett. 2004, 6, 985-987; b) Narayan, S.; Seelhammer, T.; Gawley, R. E. Tetrahedron Lett. 2004, 45, 757-759; c) Moore, J. E.; Spinks, D.; Harrity, J. P. A. Tetrahedron Lett. 2004, 45, 3189-3191; d) Meciarova, M.; Podlesna, J.; Toma, S. Monatsh. Chem. 2004, 135, 419-423; e) Wu, T. Y. H.; Schultz, P. G.; Ding, S. Org. Lett. 2003, 5, 3587-3590; f) Shi, L.; Wang, M.; Fan, C.-A.; Zhang, F. M.; Tu, Y.-Q. Org. Lett. 2003, 5, 3515-3517; g) Luo, G.; Chen. L.; Poindexter, G. S. Tetrahedron Lett. 2002, 43, 5739-5742; h) Kim, K.; Wang, B. Chem. Commun. 2001, 2268-2269.

8. a) Keyser, G. E.; Bryant, J. D.; Barrio, J. R. Tetrahedron Lett. 1979, 35, 3263-3264; b) Anton, D. L.; Korant, B. D.; Wang, C. L. J. US Pat. 4803272, 1989 [Chem. Abstr. 1989, 111, 97692]; c) Barrio, J. R.; Bryant, J. D.; Keyser, G. E. J. Med. Chem. 1980, 23, 572-574.

9. a) Gray, N. S.; Kwon, S.; Schultz, P. G. Tetrahedron Lett. 1997, 38, 1161-1164; b) Mutai, K.; Gruber, B. A.; Leonard, N. J. J. Am. Chem. Soc. 1975, 97, 4095-4104; c) Lettre, H.; Ballweg, H.; Liebigs Ann. Chem. 1960, 171-184.

10. Taha, M. L.; Lazrek, H. B. Bull. Soc. Chim. Belg. 1995, 104, 591-594.

11. a) Robins, M. J.; Hatfield, P. W.; Balzarini, J.; De Clercq, E. J. Med. Chem. 1984, 27, 1486-1492;

b) Robins, M. J.; Hatfield, P. W. Can. J. Chem. 1982, 60, 547-553.

12. Still, W. C.; Khan, M.; Mitra, A. J. Org. Chem. 1978, 43, 2923-2925.

Samples Availability: Available from the authors.

(C) 2005 by MDPI (http://www.mdpi.org). Reproduction is permitted for noncommercial purposes. 